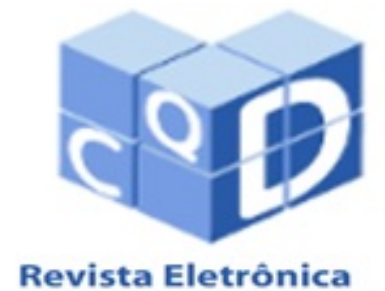

Revista Eletrônica
Paulista de Matemática

ISSN 2316-9664

Volume 15, jul. 2019

João Plínio Juchem Neto

Faculdade de Ciências

Econômicas

Universidade Federal do Rio

Grande do Sul

plinio.juchem@ufrgs.br

\section{Problema de localização de Weber com regiões circulares e funções demanda radialmente simétricas}

Weber locational problem with circular regions and radially symmetric demand functions

\begin{abstract}
Resumo
Neste trabalho generalizamos o problema de localização de Weber com firma pontual e regiões de demanda circulares a fim de considerar funções demanda radialmente simétricas dentro de cada região. Além disso, derivamos fórmulas integrais em coordenadas polares para o método iterativo de Weiszfeld modificado e o implementamos computacionalmente para a resolução numérica do problema de localização. As principais conclusões obtidas são que o método iterativo proposto converge em relativamente poucas iterações para a posição ótima em variadas configurações do problema de localização considerado, inclusive quando há regiões sobrepostas umas às outras, e que quanto mais concentrada no centro dos discos estiver a demanda, menor é o custo total mínimo associado à localização ótima.
\end{abstract}

Palavras-chave: Problema de Weber. Teoria da localização ótima. Pesquisa operacional. Regiões circulares. Método de Weiszfeld.

\begin{abstract}
In this work we generalize the discrete facility and circular demand regions Weber location problem in order to consider radially symmetric demand functions inside each region. Moreover, we derive integral formulas in polar coordinates for the modified Weiszfeld iterative method, and computationally implement it to the numerical resolution of the location problem. The main conclusions obtained are that the proposed iterative method converges in relatively few iterations to the optimal position in the various configurations of the location problem considered, even considering overlapping regions, and that the more concentrated the demand is at the center of the disks, the lower is the minimum total cost associated with the optimum location.
\end{abstract}

Keywords: Weber problem. Optimal location theory. Operational research. Circular regions. Weiszfeld method. 


\section{Introdução}

O problema de localização industrial proposto por Alfred Weber no início do século XX (WEBER, 1929), pode ser enunciado da seguinte forma (BECKMANN e THISSE, 1986; MCCANN, 2013): uma nova empresa, maximizadora de lucros e tomadora de preços nos mercados de produto, insumos, fatores de produção e transporte, precisa determinar sua localização ótima, considerando que já existe um total de $n$ fornecedores e mercados consumidores pontuais, cujas localizações, distintas, são dadas por $\vec{x}_{i}=\left(x_{i}, y_{i}\right) \in \mathbb{R}^{2}, i=1, \ldots, n$, e que o espaço é homogêneo, ou seja, as atividades da nova empresa não dependem de sua localização ${ }^{1}$. Conforme abordado mais detalhadamente na próxima seção, este problema se reduz ao problema de encontrar a localização ótima no plano, $\vec{s}^{*}$, que minimiza o custo total de transporte de insumos/mercadorias, $\min _{\vec{s} \in \mathbb{R}^{2}} C(\vec{s})=\sum_{i=1}^{n} w_{i}|| \vec{x}_{i}-\vec{s}||$, onde $w_{i}\left\|\vec{x}_{i}-\vec{s}\right\|$ é o custo de transporte de insumos/mercadorias entre a firma e o fornecedor/mercado localizado em $\vec{x}_{i}$.

Geometricamente, este problema é simplesmente o de encontrar o ponto no plano que minimiza a soma das distâncias ponderadas por $w_{i}>0$ aos $n$ pontos dados, o qual é uma generalização de um problema mais antigo proposto por Fermat no início do século XVII - encontrar o ponto no plano que minimiza a soma das distâncias a três pontos dados -, e que foi resolvido por Torricelli antes do ano de 1640 (KUHN, 1967, 1973). Numericamente, Weiszfeld foi o primeiro a propor um método iterativo de solução para o problema de Weber (WEISZFELD, 1937), o qual é analisado com mais rigor quanto a questões de convergência por Brimberg (2003) e Kuhn (1973).

Uma característica do problema de Weber é que tanto a empresa que está decidindo sua localização, quanto os fornecedores/mercados cujas localizações são dadas, são pontos discretos no plano, sem extensão espacial. Entretanto, na literatura encontramos vários trabalhos que consideram firma/fornecedores/mercados descritos por regiões extensas no plano. Love (1972) propõe um procedimento computacional para resolver o caso em que a firma é pontual, mas as demandas são regiões retangulares no plano; Drezner (1986) considera o caso em que tanto a nova firma, quanto as demandas, são descritas por regiões circulares, com as demandas dadas por densidades de probabilidade uniforme em cada região; Koshizuka e Kurita (1991) derivam fórmulas aproximadas para a distância média entre regiões circulares e as aplica na solução de problemas de localização, inclusive considerando dados regionais de quatro distritos de Tóquio, obtendo assim uma boa aproximação para a solução destes problemas; Carrizosa, Conde, Muñoz-Marquez e Puerto (1995) consideram firmas e demandas dadas por regiões compactas, descritas por medidas de probabilidade genéricas, e propõe uma notação que permite a integração de problemas discretos, regionais e mistos, provando várias propriedades que permitem a solução destes problemas por algoritmos numéricos existentes; e por fim, Chen (2001) considera o caso de uma firma pontual servindo regiões circulares/pontuais/mistas de demanda, estas últimas descritas por densidades de demanda constantes dentro de cada círculo, os quais podem ser sobrepostos, e propõe um método iterativo de Weiszfeld modificado para a resolução de problemas de localização associados.

O objetivo deste artigo é generalizar o problema considerado em Chen (2001), no sentido de considerar densidades de demanda radialmente simétricas nas regiões circulares. Em particular, adaptamos o método iterativo proposto a fim de considerar tais funções de demanda não constantes, e o aplicamos na resolução de alguns problemas de localização.

O presente artigo está estruturado da seguinte forma: na seção 2 revisamos o problema clássico de localização de Weber, sua interpretação física, o método iterativo de Weiszfeld e a

\footnotetext{
${ }^{1} \mathrm{~A}$ atividade de uma empresa dependeria de sua localização se, por exemplo, se tratasse de uma empresa exploradora de recursos naturais localizados em dado ponto do espaço, tal como uma mineradora.
} 
resolução numérica de um exemplo; na seção 3 apresentamos o problema de Weber com uma firma pontual e demandas descritas por regiões circulares com densidades de demanda radialmente simétricas, o método de Weiszfeld modificado e ilustramos com a solução de algumas configurações do problema de localização; por fim, na seção 4 finalizamos com as conclusões do trabalho e perspectivas de pesquisas futuras.

\section{Problema de localização de Weber}

Nesta seção apresentamos o problema de localização de Weber, onde uma firma pontual precisa determinar sua localização ótima, considerando que já existem um total de $n$ fornecedores e mercados consumidores pontuais, cujas localizações, distintas, são dadas por $\vec{x}_{i}=\left(x_{i}, y_{i}\right) \in \mathbb{R}^{2}$, $i=1, \ldots, n$. Inicialmente definimos:

- $q\left(\vec{x}_{i}\right)$ como a quantidade de produto final vendido pela nova empresa para o mercado consumidor localizado em $\vec{x}_{i}$ e $t^{i}$ a sua taxa de transporte;

- $q_{1}\left(\vec{x}_{i}\right), \ldots, q_{m}\left(\vec{x}_{i}\right)$ como as quantidades dos $m$ insumos vendidos pelo fornecedor localizado em $\vec{x}_{i}$ à nova empresa e $t_{1}^{i}, \ldots, t_{m}^{i}$ as suas respectivas taxas de transporte.

As quantidades $q\left(\vec{x}_{i}\right), q_{1}\left(\vec{x}_{i}\right), \ldots, q_{m}\left(\vec{x}_{i}\right)$ são não-negativas, não podendo todas serem simultaneamente nulas. Geralmente essas quantidades são medidas em unidades de peso (Kg, ton) e as taxas de transporte associadas como unidade monetária (\$) por peso vezes distância $(\$ /(\mathrm{Kg} . \mathrm{Km})$, $\$ /($ ton. $\mathrm{Km})$, etc.). Sendo assim, temos que o custo total (em $\$$ ) para transportar bens entre a nova empresa e o fornecedor/mercado localizado em $\vec{x}_{i}$ é dado por:

$$
C_{i}(\vec{s})=\underbrace{\left(t^{i} q\left(\vec{x}_{i}\right)+\sum_{j=1}^{m} t_{j}^{i} q_{j}\left(\vec{x}_{i}\right)\right)}_{=w_{i}} d_{i}=w_{i} d_{i},
$$

onde $\vec{s}=(x, y) \in \mathbb{R}^{2}$ é a localização da nova empresa, $w_{i}>0$ é definido como o peso ideal associado à localização $\vec{x}_{i}$ e $d_{i}$ é a distância entre esta localização e a nova empresa. Geralmente essa distância é calculada através da norma euclidiana:

$$
d_{i}=d\left(\vec{x}_{i}, \vec{s}\right)=\left\|\vec{x}_{i}-\vec{s}\right\|=\sqrt{\left(x_{i}-x\right)^{2}+\left(y_{i}-y\right)^{2}} .
$$

Note que o peso ideal $w_{i}$ é uma função crescente das quantidades de bens transacionadas entre a nova empresa e a posição $\vec{x}_{i}$ e das taxas de transporte destes bens.

Como a empresa não consegue influenciar os preços de mercado, para atingir o objetivo de maximizar seu lucro, ela deve, de forma equivalente, encontrar a localização que minimiza o custo total de transporte:

$$
C(\vec{s})=\sum_{i=1}^{n} C_{i}(\vec{s}),
$$

isto é, precisa resolver o problema de minimização:

$$
\min _{\vec{s} \in \mathbb{R}^{2}} C(\vec{s})=\sum_{i=1}^{n} w_{i} \mid\left\|\vec{x}_{i}-\vec{s}\right\| .
$$


No geral, como a norma euclidiana é uma função convexa no plano e $w_{i}>0$, então a função custo total do problema acima também é convexa. Desta forma, existe um minimizador global $\vec{s}^{*}$ para o custo total de transporte, o qual não precisa único. Entretanto, Kuhn (1967) apresenta os seguintes resultados:

Teorema 1: Se os $n$ pontos distintos $\vec{x}_{i}=\left(x_{i}, y_{i}\right) \in \mathbb{R}^{2}$ não são colineares, então $C(\vec{s})$ é uma função estritamente convexa de $\vec{s}$. Se os pontos são colineares, então $C(\vec{s})$ é linear por partes nas retas conectando estes pontos, e estritamente convexa no resto do plano.

Corolário 1: Se os $n$ pontos distintos $\vec{x}_{i}=\left(x_{i}, y_{i}\right) \in \mathbb{R}^{2}$ não são colineares, então $C(\vec{s})$ possui um único ponto de mínimo global.

Sendo assim, um ponto que satisfaça as condições de primeira ordem abaixo é automaticamente um ponto de mínimo global de $C(\vec{s})$ :

$$
\begin{aligned}
& \frac{\partial C}{\partial x}=\sum_{i=1}^{n} \frac{w_{i}\left(x-x_{i}\right)}{\left\|\vec{x}_{i}-\vec{s}\right\|}=0, \\
& \frac{\partial C}{\partial y}=\sum_{i=1}^{n} \frac{w_{i}\left(y-y_{i}\right)}{\left\|\vec{x}_{i}-\vec{s}\right\|}=0 .
\end{aligned}
$$

Em notação vetorial, (2a)-(2b) podem ser escritas como:

$$
\vec{\nabla} C(\vec{s})=\sum_{i=1}^{n} \frac{w_{i}\left(\vec{s}-\vec{x}_{i}\right)}{\left\|\vec{x}_{i}-\vec{s}\right\|}=\overrightarrow{0},
$$

o que é válido sempre que $\vec{s} \neq \vec{x}_{i}, i=1, \ldots, n$. Da expressão acima temos que:

$$
\vec{s}=\frac{\sum_{i=1}^{n} \frac{w_{i} \vec{x}_{i}}{\left\|\vec{x}_{i}-\vec{s}\right\|}}{\sum_{i=1}^{n} \frac{w_{i}}{\left\|\vec{x}_{i}-\vec{s}\right\|}} .
$$

Embora não seja possível resolver explicitamente esta equação para $\vec{s}$, podemos utilizá-la para definir, de forma bastante natural, um método numérico iterativo. Substituindo o $\vec{s}$ no lado direito desta equação por $\vec{s}_{k}$, a posição da nova empresa no passo atual $k$, e o do lado esquerdo por $\vec{s}_{k+1}$, sua posição no passo seguinte, $k+1$, e considerando $\vec{s}_{0}$ uma posição inicial qualquer da nova empresa que não coincida com nenhum $\vec{x}_{i}$, obtemos:

$$
\vec{s}_{k+1}=\frac{\sum_{i=1}^{n} \frac{w_{i} \vec{x}_{i}}{\left\|\vec{x}_{i}-\vec{s}_{k}\right\|}}{\sum_{i=1}^{n} \frac{w_{i}}{\left\|\vec{x}_{i}-\vec{s}_{k}\right\|}}, k=0,1,2,3, \ldots
$$

A aplicação sucessiva desta fórmula fornece uma sequência de aproximações da solução de (3) que esperamos convergir para a posição ótima $\vec{s}^{*}$ :

$$
\vec{s}_{1}, \vec{s}_{2}, \vec{s}_{3}, \cdots \rightarrow \vec{s}^{*}
$$

Na prática, a iteração é executada até que certo critério de parada seja satisfeito. Uma possibilidade é parar quando o erro relativo estimado entre duas aproximações sucessivas fique abaixo de um valor pequeno $0<\varepsilon<<1$ definido a priori:

$$
\frac{\| \vec{s}_{k+1}-\vec{s}_{k}||}{\left\|\vec{s}_{k}\right\|}<\varepsilon .
$$


Observe que (4) é na verdade uma versão do método do gradiente, pois pode ser reescrito como:

$$
\vec{s}_{k+1}=\vec{s}_{k}-\alpha_{k} \vec{\nabla} C\left(\vec{s}_{k}\right), k=0,1,2,3, \ldots
$$

sendo o tamanho do passo dado por:

$$
\alpha_{k}=\frac{1}{\sum_{i=1}^{n} \frac{w_{i}}{\left\|\vec{x}_{i}-\vec{s}_{k}\right\|}} .
$$

Observação 1: Weiszfeld foi o primeiro autor a propor o método numérico iterativo apresentado acima, a fim de resolver o problema de Weber (WEISZFELD, 1937). Kuhn (1973) demonstra um teorema de convergência para este método, nos casos em que o ponto $\vec{s}_{k}$ não coincida, em nenhum passo de tempo, com as localizações dos fornecedores/mercados dados, isto é, desde que $\vec{s}_{k} \neq \vec{x}_{i}, i=1, \ldots, n$, para todo passo $k=0,1,2,3, \ldots$ É importante notar, entretanto, que existem condições iniciais, $\vec{s}_{0}$, que fazem com que o método iterativo gere $\vec{s}_{k}=\vec{x}_{i}$ para algum $k$ e $i$, causando a divergência do mesmo. Entretanto, tais condições iniciais problemáticas são relativamente raras - para uma discussão mais detalhada, ver Brimberg (2003)

Exemplo 1: Na Figura 1 apresentamos a solução do modelo de Weber considerando três localidades dadas pelos vértices de um triângulo equilátero, através da aplicação do método iterativo (4)-(5). O mesmo foi implementado no software matemático MATLAB ${ }^{\circledR}$. Neste exemplo são considerados quatro cenários para os pesos ideais, sendo que os parâmetros do problema são apresentados na Tabela 1.

Tabela 1 - Parâmetros do problema considerado no Exemplo 1

\begin{tabular}{|c|c|}
\hline$\vec{x}_{1}$ & $(100,100)$ \\
\hline$\vec{x}_{2}$ & $(900,100)$ \\
\hline$\vec{x}_{3}$ & $\left(500,100+\sqrt{800^{2}-400^{2}}\right)$ \\
\hline$\vec{s}_{0}$ & $(150,200)$ \\
\hline$\varepsilon$ & $10^{-6}$ \\
\hline
\end{tabular}

No primeiro cenário (A), os pesos ideais são iguais e unitários para as três localidades, $w_{1}=$ $w_{2}=w_{3}=1$. Neste caso, a localização ótima da nova firma $s^{*}$, destacada em vermelho, fica equidistante às três localidades dadas. O rastro em azul, partindo de $\vec{s}_{0}$, representa os passos intermediários do método numérico. O segundo cenário (B) retrata um aumento de $50 \%$ no peso ideal associado à posição $\vec{x}_{2}, w_{1}=1, w_{2}=1.5, w_{3}=1$. Tal aumento poderia ser o resultado de um aumento nas quantidades de bens transacionados com esta região e/ou em suas taxas de transporte. Neste caso, como podemos observar na figura, a localização ótima se aproxima de $\vec{x}_{2}$, pois a única forma da nova firma compensar este aumento em $w_{2}$ é diminuindo sua distância em relação a $\vec{x}_{2}$. No terceiro cenário $(\mathrm{C}), w_{3}$ aumenta em relação aos outros pesos $\left(w_{1}=1, w_{2}=\right.$ $1.5, w_{3}=2$ ), de forma que seu peso ideal se torna o maior. Como consequência, a posição ótima se aproxima de $\vec{x}_{3}$. Por fim, no último caso (D), $w_{1}$ aumenta em relação aos outros pesos ideais $\left(w_{1}=2.5, w_{2}=1.5, w_{3}=2\right)$, de forma a se tornar o maior peso. Por sua vez, a posição ótima se aproxima de $\vec{x}_{1}$. Na Tabela 2 apresentamos os valores ótimos retornados pelo método numérico 
para cada um dos cenários representados na figura. O número de iterações numéricas necessárias para a convergência é apresentado na última linha desta tabela.

Em suma, uma nova firma maximizadora de lucros e tomadora de preços tenderá a se localizar mais próxima do local com o qual transaciona mais intensamente e cujas taxas de transportes sejam mais altas
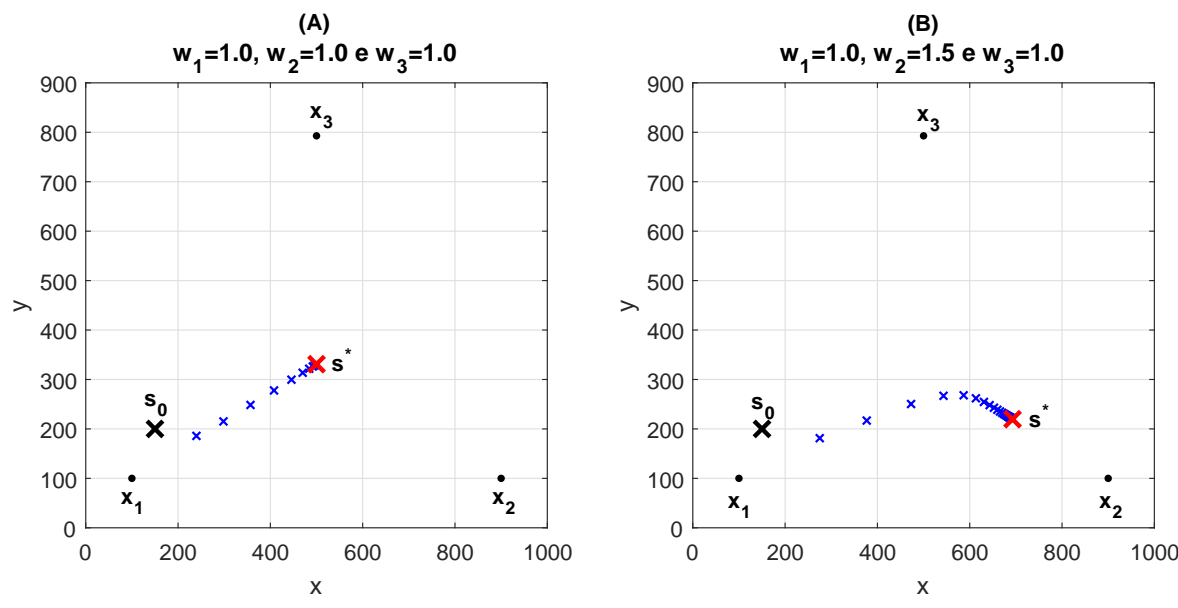

(C)
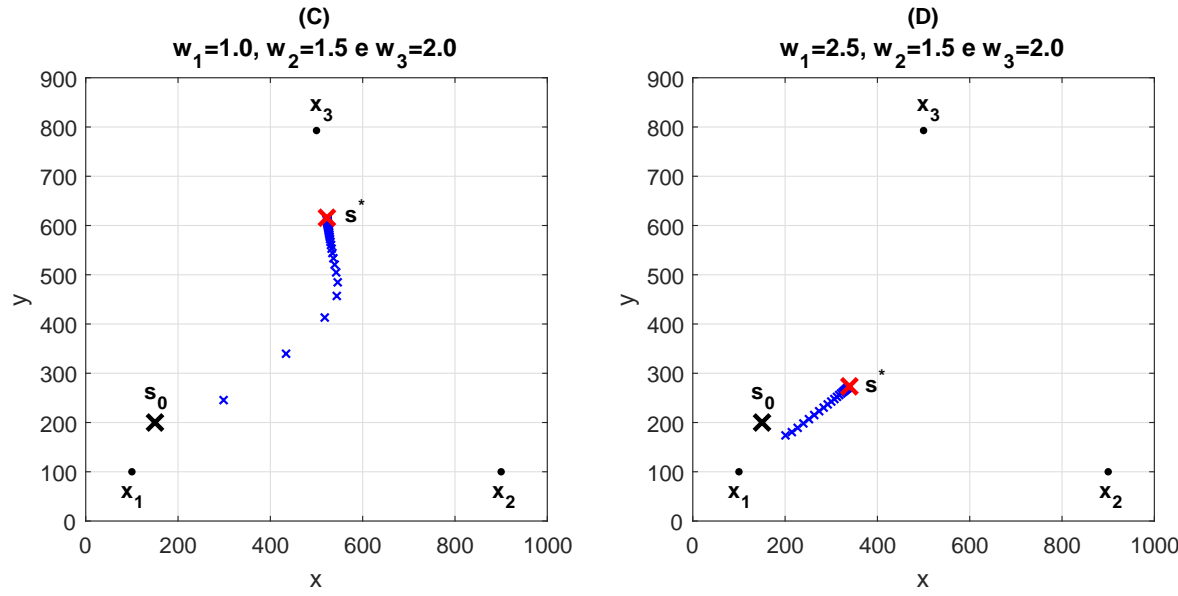

Figura 1 - Solução do problema de Weber apresentado no Exemplo 1 Fonte: Elaborado pelo autor

Interpretação Física: Em Chen (2001) é apresentada uma interpretação física para o problema de localização de Weber, onde o fornecedor/mercado localizado em $\vec{x}_{i}$ exerce uma força de atração sobre a nova empresa, força esta que possui magnitude dada pelo seu peso ideal. Para ver isto, basta considerar a função custo $C_{i}(\vec{s})$ como uma função potencial e suas derivadas parciais como definidoras de um campo de forças dado pelo campo vetorial gradiente:

$$
\vec{F}_{i}=-\vec{\nabla} C_{i}=\left(w_{i} \frac{x_{i}-x}{d_{i}}, w_{i} \frac{y_{i}-y}{d_{i}}\right)=\left(w_{i} \cos \phi_{i}, w_{i} \operatorname{sen} \phi_{i}\right),
$$

onde $\phi_{i}$ é o ângulo entre a reta ligando $\vec{s}$ e $\vec{x}_{i}$ e a horizontal, conforme apresentado na Figura 2 . O 
Tabela 2 - Valores ótimos do exemplo com $n=3$

\begin{tabular}{|c|c|c|c|c|}
\hline & $(\mathrm{A})$ & $(\mathrm{B})$ & $(\mathrm{C})$ & $(\mathrm{D})$ \\
\hline$\vec{s}^{*}$ & $(500,00 ; 330,94)$ & $(692,79 ; 219,63)$ & $(522,51 ; 615,78)$ & $(339,31 ; 273,48)$ \\
\hline$C\left(\vec{s}^{*}\right)$ & $1.385,64$ & 1.568 .38 & $1.982,41$ & $2.706,57$ \\
\hline$d_{1}$ & 461,88 & 604,74 & 666,74 & 295,58 \\
\hline$d_{2}$ & 461,88 & 239,27 & 639,16 & 586,91 \\
\hline$d_{3}$ & 461,88 & 604,74 & 178,47 & 543,63 \\
\hline Iterações & 23 & 66 & 104 & 59 \\
\hline
\end{tabular}

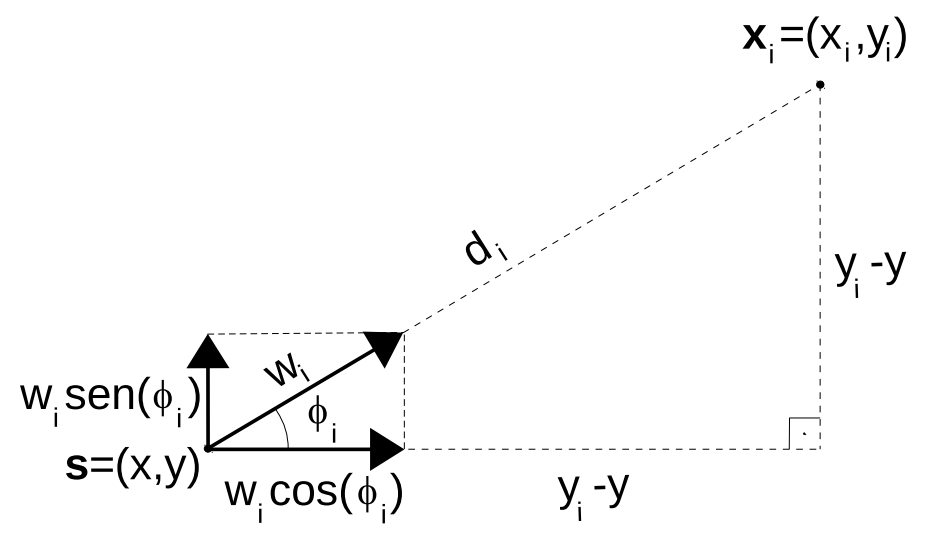

Figura 2 - Definição do ângulo $\phi_{i}$ Fonte: Adaptado de Chen (2001)

campo de atração de um fornecedor/mercado localizado em $\vec{x}_{i}=\overrightarrow{0}$ é ilustrado na Figura 3. Note que a magnitude da força atrativa na direção de $\vec{x}_{i}$ é dada pelo peso ideal $w_{i}$ e não depende da distância ao fornecedor/mercado.

Assim, a força resultante sobre a nova empresa, $\vec{F}$, é dada pela soma vetorial das forças atrativas $\operatorname{dos} n$ fornecedores/mercados:

$$
\vec{F}=\sum_{i=1}^{n} \vec{F}_{i}=-\sum_{i=1}^{n} \vec{\nabla} C_{i}=-\nabla\left(\sum_{i=1}^{n} \vec{C}_{i}\right)=-\vec{\nabla} C .
$$

Portanto, nesta nova linguagem, a localização ótima de Weber será o ponto do plano, $\vec{s}^{*}$, onde as forças atrativas dos $n$ fornecedores/mercados entram em equilíbrio, ou seja, onde a força resultante sobre a nova empresa é nula, $\vec{F}\left(\vec{s}^{*}\right)=\overrightarrow{0} \square$

\section{Modelo de Weber com mercados circulares e demandas ra- dialmente simétricas}

Aqui introduzimos uma generalização do problema de Weber com regiões circulares e demandas uniformemente distribuídas considerado em Chen (2001). Ainda consideramos uma nova empresa pontual, mas agora as $n$ regiões circulares descrevendo mercados/fornecedores são dadas 


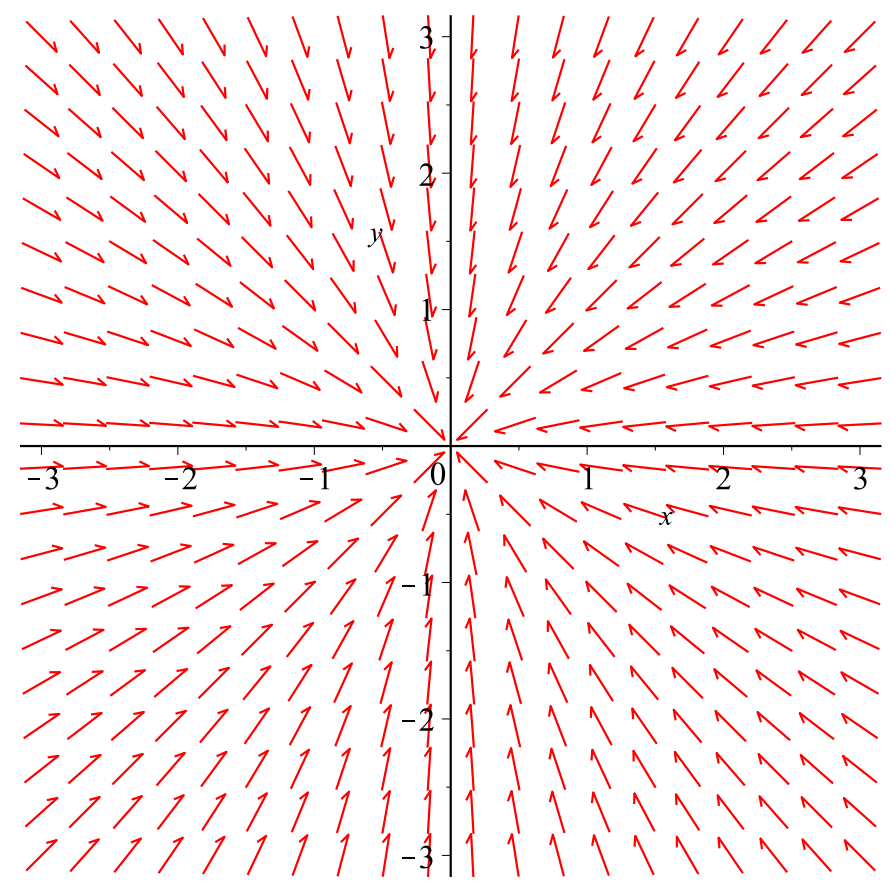

Figura 3 - Campo de atração de um fornecedor/mercado localizado em $\vec{x}_{i}=\overrightarrow{0}$

Fonte: Elaborado pelo autor

por discos fechados no plano $D_{i}$, de raio $R_{i}$ e centro $\vec{x}_{i}$, cujos pesos ideais são funções densidade radialmente simétricas integráveis em $D_{i}$ dadas por $\omega_{i}: D_{i} \rightarrow \mathbb{R}_{+}, i=1, \ldots, n$. Sem perda de generalidade, vamos considerar $D_{i}$ um mercado consumidor; assim a função $\omega_{i}(\vec{z}), \vec{z}=\left(z_{x}, z_{y}\right) \in D_{i}$, pode ser interpretada como uma função densidade de demanda relacionada a esta região.

Nesse caso, o custo de transporte de bens entre a nova empresa localizada em $\vec{s}$ e a região $D_{i}$ é dado pela seguinte integral dupla:

$$
C_{i}(\vec{s})=\iint_{D_{i}} \omega_{i}(\vec{z}) d(\vec{z}, \vec{s}) d A .
$$

Observe que se $\iint_{D_{i}} \omega_{i}(\vec{z}) d A=1, \omega_{i}(\vec{z})$ pode ser interpretada como uma função densidade de probabilidade, e portanto $C_{i}(\vec{s})$ pode ser vista como a distância esperada entre a nova empresa e a região $D_{i}$ (CARRIZOSA, CONDE, MUÑOZ-MARQUEZ e PUERTO, 1995). Considerando o Teorema 3.2 demonstrado em Carrizosa, Conde, Muñoz-Marquez e Puerto (1995), temos que (6) é uma função estritamente convexa para uma ampla gama de funções $\omega_{i}(\vec{z})$. Desta forma, o problema de minimizar os custos totais de transporte:

$$
\min _{\vec{s}} C(\vec{s})=\sum_{i=1}^{n} \iint_{D_{i}} \omega_{i}(\vec{z})|| \vec{z}-\vec{s} \| d A
$$

possui minimizador global único. As condições de primeira ordem para este problema são:

$$
\begin{aligned}
& \frac{\partial C}{\partial x}=\sum_{i=1}^{n} \iint_{D_{i}} \frac{\omega_{i}(\vec{z})\left(x-z_{x}\right)}{\|\vec{z}-\vec{s}\|} d A=0, \\
& \frac{\partial C}{\partial y}=\sum_{i=1}^{n} \iint_{D_{i}} \frac{\omega_{i}(\vec{z})\left(y-z_{y}\right)}{\|\vec{z}-\vec{s}\|} d A=0,
\end{aligned}
$$


as quais podem ser combinadas como:

$$
\vec{\nabla} C(\vec{s})=\sum_{i=1}^{n} \iint_{D_{i}} \frac{\omega_{i}(\vec{z})(\vec{s}-\vec{z})}{\|\vec{z}-\vec{s}\|} d A=\overrightarrow{0}
$$

Observação 2: Considerando o caso particular em que a função demanda é concentrada na origem:

$$
\omega=\omega_{i}(\vec{z}) \equiv w_{i} \delta\left(x-x_{i}, y-y_{i}\right),
$$

onde $w_{i}$ é a demanda total associada à região $D_{i}$ e $\delta\left(x-x_{i}, y-y_{i}\right)$ é a função delta de Dirac centrada em $\vec{x}_{i}$, temos, utilizando a propriedade da filtragem ${ }^{2}$, que a equação (7) se reduz ao problema de Weber original (1), com a condição de primeira ordem (9) se reduzindo a (3)

De forma análoga à seção anterior, (9) pode ser utilizada para definir o seguinte método iterativo, adaptação do método de Weiszfeld da seção anterior:

$$
\vec{s}_{k+1}=\frac{\sum_{i=1}^{n} \iint_{D_{i}} \frac{\omega_{i}(\vec{z}) \vec{z}}{\left\|\vec{z}-\vec{s}_{k}\right\|} d A}{\sum_{i=1}^{n} \iint_{D_{i}} \frac{\omega_{i}(\vec{z})}{\left\|\vec{z}-\vec{s}_{k}\right\|} d A}=\vec{s}_{k}-\beta_{k} \vec{\nabla} C\left(\vec{s}_{k}\right), k=0,1,2,3, \ldots
$$

onde o tamanho do passo, $\beta_{k}$ é definido como:

$$
\beta_{k}=\frac{1}{\sum_{i=1}^{n} \iint_{D_{i}} \frac{\omega_{i}(\vec{z})}{\left\|\vec{z}-\vec{s}_{k}\right\|} d A}=\frac{1}{\sum_{i=1}^{n} W_{i}}=\frac{1}{W},
$$

sendo que definimos:

$$
W_{i}=\iint_{D_{i}} \frac{\omega_{i}(\vec{z})}{\left\|\vec{z}-\vec{s}_{k}\right\|} d A \text { e } W=\sum_{i=1}^{n} W_{i}
$$

Em termos de componentes, (10) pode ser escrito da seguinte forma:

$$
\begin{aligned}
& x_{k+1}=x_{k}+\frac{1}{W} \sum_{i=1}^{n} \iint_{D_{i}} \frac{\omega_{i}(\vec{z})\left(z_{x}-x\right)}{\|\vec{z}-\vec{s}\|} d A, \\
& y_{k+1}=y_{k}+\frac{1}{W} \sum_{i=1}^{n} \iint_{D_{i}} \frac{\omega_{i}(\vec{z})\left(z_{y}-y\right)}{\|\vec{z}-\vec{s}\|} d A .
\end{aligned}
$$

Definindo $\zeta=\zeta(\vec{s})$ como o ângulo entre o segmento de reta $\overrightarrow{s z}$ e a horizontal (ver Figura 4 ), temos que:

$$
\cos \zeta=\frac{z_{x}-x}{\|\vec{z}-\vec{s}\|} \text { e sen } \zeta=\frac{z_{y}-y}{\|\vec{z}-\vec{s}\|}
$$

e assim:

$$
\begin{aligned}
& x_{k+1}=x_{k}+\frac{1}{W} \sum_{i=1}^{n} \iint_{D_{i}} \omega_{i}(\vec{z}) \cos \zeta d A, \\
& y_{k+1}=y_{k}+\frac{1}{W} \sum_{i=1}^{n} \iint_{D_{i}} \omega_{i}(\vec{z}) \operatorname{sen} \zeta d A .
\end{aligned}
$$

\footnotetext{
${ }^{2}$ Propriedade da filtragem da função delta de Dirac: $\iint_{\mathbb{R}^{2}} \delta\left(\vec{z}-\vec{x}_{i}\right) f(\vec{z}) d A=f\left(\vec{x}_{i}\right)$.
} 


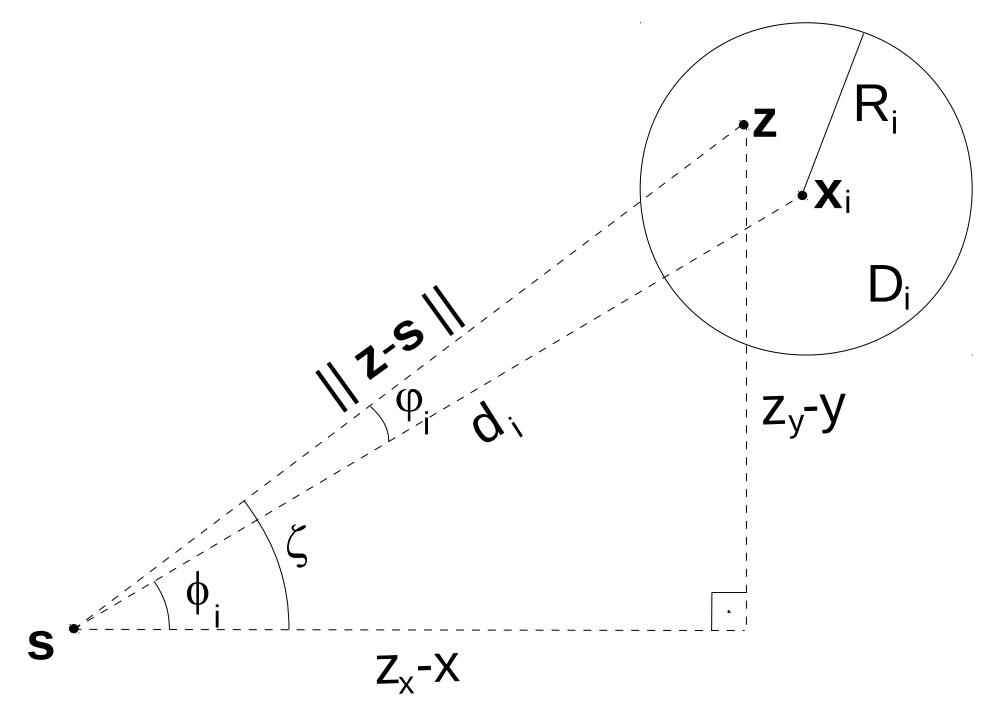

Figura 4 - Definição dos ângulos $\zeta$ e $\varphi_{i}$

Fonte: Adaptado de Chen (2001)

Na Figura 4 também definimos o ângulo entre a reta $\overrightarrow{s z}$ e a reta $\overrightarrow{s x}_{i}$ como $\varphi_{i}=\zeta-\phi_{i}$, de onde temos que:

$$
\begin{aligned}
& \cos \zeta=\cos \left(\phi_{i}+\varphi_{i}\right)=\cos \phi_{i} \cos \varphi_{i}-\operatorname{sen} \phi_{i} \operatorname{sen} \varphi_{i}, \\
& \operatorname{sen} \zeta=\operatorname{sen}\left(\phi_{i}+\varphi_{i}\right)=\operatorname{sen} \phi_{i} \cos \varphi_{i}+\cos \phi_{i} \operatorname{sen} \varphi_{i}
\end{aligned}
$$

Note que, para percorrer todo o disco $D_{i}$, o ângulo $\varphi_{i}$ deve percorrer o intervalo simétrico:

$$
\left[\varphi_{i}^{\min }, \varphi_{i}^{\max }\right]=\left\{\begin{array}{l}
{\left[-\operatorname{arcsen}\left(\frac{R_{i}}{d_{i}}\right), \operatorname{arcsen}\left(\frac{R_{i}}{d_{i}}\right)\right], \text { se } d_{i} \geq R_{i}} \\
{[-\pi, \pi], \text { se } d_{i}<R_{i}}
\end{array}\right.
$$

onde $d_{i}$ é a distância entre $\vec{s}$ e o centro do disco $D_{i}, \vec{x}_{i}$.

Como as funções densidade de demanda são radialmente simétricas em cada disco, temos que as integrais em (11a)-(11b) podem ser simplificadas:

$$
\begin{aligned}
\iint_{D_{i}} \omega_{i}(\vec{z}) \cos \zeta d A & =\underbrace{\left(\iint_{D_{i}} \omega_{i}(\vec{z}) \cos \varphi_{i} d A\right)}_{=I_{i}} \cos \phi_{i}-\underbrace{\left(\iint_{D_{i}} \omega_{i}(\vec{z}) \operatorname{sen} \varphi_{i} d A\right)}_{=0 \text { por simetria }} \operatorname{sen} \phi_{i} \\
\iint_{D_{i}} \omega_{i}(\vec{z}) \operatorname{sen} \zeta d A & =\underbrace{\left(\iint_{D_{i}} \omega_{i}(\vec{z}) \operatorname{sen} \varphi_{i} d A\right)}_{=0 \text { por simetria }} \cos \phi_{i}+\underbrace{\left(\iint_{D_{i}} \omega_{i}(\vec{z}) \cos \varphi_{i} d A\right)}_{=I_{i}} \operatorname{sen} \phi_{i}
\end{aligned}
$$

e portanto, o método iterativo se reduz a:

$$
\begin{aligned}
& x_{k+1}=x_{k}+\frac{\sum_{i=1}^{n} I_{i} \cos \phi_{i}}{W} \\
& y_{k+1}=y_{k}+\frac{\sum_{i=1}^{n} I_{i} \operatorname{sen} \phi_{i}}{W}, k=0,1,2,3, \ldots
\end{aligned}
$$


onde definimos $I_{i}=\iint_{D_{i}} \omega_{i}(\vec{z}) \cos \varphi_{i} d A$ como a magnitude total da força de atração exercida por todos os pontos do disco $D_{i}$, projetados na direção da reta conectando $\vec{s}$ ao centro do disco, $\vec{x}_{i}$, que é seu centro de massa.

As fórmulas derivadas acima são válidas para $\vec{s}$ estando dentro ou fora do disco $D_{i}$. Considerando coordenadas polares, temos que:

$$
I_{i}=\int_{\varphi_{i}^{\min }}^{\varphi_{i}^{\max }} \int_{r_{i}^{\min }\left(\varphi_{i}\right)}^{r_{i}^{\max }\left(\varphi_{i}\right)} \omega_{i}(r) \cos \varphi_{i} r d r d \varphi_{i}
$$

onde $\varphi_{i}^{\min }$ e $\varphi_{i}^{\max }$ são dados por (12), $r_{i}^{\text {min }}$ é dado por:

$$
r_{i}^{\min }\left(\varphi_{i}\right)=\left\{\begin{array}{l}
d_{i} \cos \varphi_{i}-\sqrt{R_{i}^{2}-d_{i}^{2} \operatorname{sen}^{2} \varphi_{i}}, \text { se } d_{i} \geq R_{i} \\
0, \text { se } d_{i}<R_{i}
\end{array}\right.
$$

e $r_{i}^{\max }$ por:

$$
r_{i}^{\max }\left(\varphi_{i}\right)=d_{i} \cos \varphi_{i}+\sqrt{R_{i}^{2}-d_{i}^{2} \operatorname{sen}^{2} \varphi_{i}}, \forall d_{i},
$$

Os detalhes do cálculo de $r_{i}^{\min }$ e $r_{i}^{\max }$, que decorrem de simples considerações geométricas, podem ser encontrados em Chen (2001). Além disso, temos que:

$$
C_{i}=\int_{\varphi_{i}^{\min }}^{\varphi_{i}^{\max }} \int_{r_{i}^{\min }\left(\varphi_{i}\right)}^{r_{i}^{\max }\left(\varphi_{i}\right)} \omega_{i}(r) r^{2} d r d \varphi_{i}
$$

e:

$$
W_{i}=\int_{\varphi_{i}^{\min }}^{\varphi_{i}^{\max }} \int_{r_{i}^{\min }\left(\varphi_{i}\right)}^{r_{i}^{\max }\left(\varphi_{i}\right)} \omega_{i}(r) d r d \varphi_{i}
$$

onde os limites de integração também são dados por (12), (15) e (16).

Observação 3: Se considerarmos funções demanda constantes no método iterativo acima (13a)(18), i.e. $\omega_{i}(r) \equiv w_{i}=$ constante, recuperamos as fórmulas apresentadas em Chen (2001)

A seguir apresentamos alguns exemplos de funções peso ideais radialmente simétricas, com a maior densidade de demanda no centro do disco:

(a) Função pontual: é o caso considerado no modelo original de Weber, onde toda a demanda $w_{i}$ está concentrada no centro do disco, o que pode ser descrito pela função delta de Dirac:

$$
\omega_{i}(\vec{z}) \equiv w_{i} \delta\left(x-x_{i}, y-y_{i}\right)
$$

(b) Função gaussiana: neste caso o disco apresenta demanda no formato de uma gaussiana radialmente simétrica com desvio padrão $\sigma$, com a máxima densidade de demanda em seu centro, e então decaindo exponencialmente até a fronteira:

$$
\omega_{i}(\vec{z})=w_{i} \frac{\exp \left[-\frac{\left(x-x_{i}\right)^{2}}{2 \sigma^{2}}-\frac{\left(y-y_{i}\right)^{2}}{2 \sigma^{2}}\right]}{\iint_{D_{i}} \exp \left[-\frac{\left(x-x_{i}\right)^{2}}{2 \sigma^{2}}-\frac{\left(y-y_{i}\right)^{2}}{2 \sigma^{2}}\right] d A} .
$$

Escolhendo $\sigma=\frac{R}{4}$, a fim de garantir uma queda na densidade de demanda nem muita rápida, nem muito lenta, até chegar na fronteira do disco, temos:

$$
\omega_{i}(\vec{z})=w_{i} \frac{\exp \left[-\frac{16\left(x-x_{i}\right)^{2}}{2 R^{2}}-\frac{16\left(y-y_{i}\right)^{2}}{2 R^{2}}\right]}{\iint_{D_{i}} \exp \left[-\frac{16\left(x-x_{i}\right)^{2}}{2 R^{2}}-\frac{16\left(y-y_{i}\right)^{2}}{2 R^{2}}\right] d A} .
$$


(c) Função cônica côncava: neste caso cada disco possui um cone côncavo de demanda, apresentando a máxima densidade de demanda em seu centro, e então decaindo linearmente até zero no círculo fronteiriço:

$$
\omega_{i}(\vec{z})=\frac{3 w_{i}}{\pi R_{i}^{2}}\left(1-\frac{1}{R_{i}} \sqrt{\left(x-x_{i}\right)^{2}+\left(y-y_{i}\right)^{2}}\right) .
$$

(d) Função paraboloide côncavo: neste caso cada disco possui um paraboloide côncavo de demanda, com a máxima densidade de demanda em seu centro, e então decaindo parabolicamente até zero na fronteira:

$$
\omega_{i}(\vec{z})=\frac{2 w_{i}}{\pi R_{i}^{2}}\left(\frac{R^{2}-\left[\left(x-x_{i}\right)^{2}+\left(y-y_{i}\right)^{2}\right]}{R_{i}^{2}}\right) .
$$

O caso uniforme, considerado em Chen (2001) é:

(e) Função constante: podemos considerar que a demanda total $w_{i}$ é uniformemente distribuída no disco $D_{i}$, ou seja:

$$
\omega_{i}(\vec{z})=\frac{w_{i}}{\pi R_{i}^{2}}
$$

Abaixo apresentamos exemplos de funções peso ideais radialmente simétricas, com maior densidade de demanda na parte fronteiriça do disco:

(f) Função gaussiana invertida: neste caso o disco apresenta demanda no formato de uma gaussiana invertida radialmente simétrica com desvio padrão $\sigma$, com demanda nula no centro e máxima densidade de demanda na fronteira:

$$
\omega_{i}(\vec{z})=w_{i} \frac{1-\exp \left[-\frac{\left(x-x_{i}\right)^{2}}{2 \sigma^{2}}-\frac{\left(y-y_{i}\right)^{2}}{2 \sigma^{2}}\right]}{\pi R^{2}-\iint_{D_{i}} \exp \left[-\frac{\left(x-x_{i}\right)^{2}}{2 \sigma^{2}}-\frac{\left(y-y_{i}\right)^{2}}{2 \sigma^{2}}\right] d A} .
$$

Escolhendo $\sigma=\frac{R}{4}$, a fim de garantir uma queda na densidade de demanda nem muita rápida, nem muito lenta, até chegar na fronteira do disco, temos:

$$
\omega_{i}(\vec{z})=w_{i} \frac{1-\exp \left[-\frac{16\left(x-x_{i}\right)^{2}}{2 R^{2}}-\frac{16\left(y-y_{i}\right)^{2}}{2 R^{2}}\right]}{\pi R^{2}-\iint_{D_{i}} \exp \left[-\frac{16\left(x-x_{i}\right)^{2}}{2 R^{2}}-\frac{16\left(y-y_{i}\right)^{2}}{2 R^{2}}\right] d A} .
$$

(g) Função cônica convexa: neste caso cada disco possui um cone convexo de demanda, apresentando demanda nula no centro, e então crescendo linearmente até o círculo fronteiriço:

$$
\omega_{i}(\vec{z})=\frac{3 w_{i}}{2 \pi R_{i}^{2}}\left(\frac{1}{R_{i}} \sqrt{\left(x-x_{i}\right)^{2}+\left(y-y_{i}\right)^{2}}\right) .
$$

(h) Função paraboloide convexo: neste caso cada disco possui um paraboloide convexo de demanda, com demanda nula no centro e crescendo parabolicamente até na fronteira:

$$
\omega_{i}(\vec{z})=\frac{2 w_{i}}{\pi R_{i}^{2}}\left[\frac{\left(x-x_{i}\right)^{2}+\left(y-y_{i}\right)^{2}}{R_{i}^{2}}\right] .
$$

Exemplo 2: Na Figura 5 (A) apresentamos os perfis das funções demanda unitárias $\left(w_{i}=1\right)$ radialmente simétricas e com densidade concentrada mais próximo ao centro do disco (19)-(23): 
demanda pontual considerada no modelo de Weber padrão e dada pela função delta de Dirac (representada pela seta vertical preta), gaussiana, cônica côncava, paraboloide côncavo e constante, todas ordenada da maior para a menor concentração no centro e consideradas em um disco de raio unitário $\left(R_{i}=1\right)$. Já na Figura 5 (B) apresentamos os perfis das funções demanda unitárias com densidade concentrada mais próxima à fronteira do disco (23)-(26): paraboloide convexo, cônica convexa, gaussiana invertida e constante, todas ordenadas da maior para a menor concentração na fronteira.
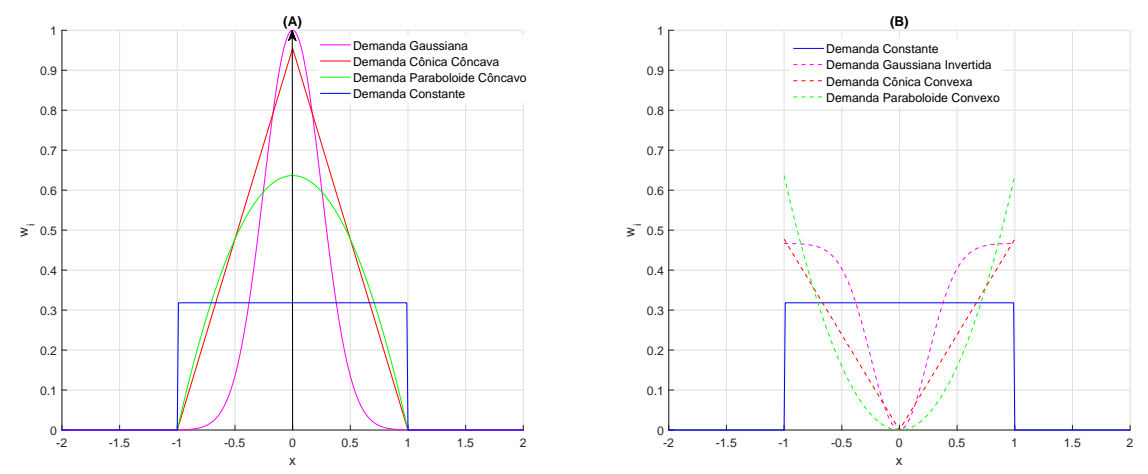

Figura 5 - Perfis das funções demanda unitárias $w_{i}=1$ do Exemplo 2 Fonte: Elaborado pelo autor

Na Figura 6, gráfico da esquerda, apresentamos a magnitude total da força de atração exercida por todos os pontos de um disco $D_{i}$ de raio unitário $R=1, I_{i}$, a qual é calculada através da integral (14), assim como o custo total associado a este disco, $C_{i}$, dado por (17), para distâncias entre $\vec{s}$ e $\vec{x}_{i}, d_{i}$, variando de 0 (centro do disco) até 3 (três vezes o raio do disco). Foram consideradas as funções demanda apresentadas anteriormente (19)-(26), todas com uma demanda total unitária, ou seja, $w_{i}=1$. As integrais duplas foram calculadas numericamente através do comando integral2 do software MATLAB ${ }^{\circledR}$. Em ordem decrescente de força atrativa, temos as demandas pontual, gaussiana, cônica côncava, paraboloide côncavo, constante, gaussiana invertida, cônica convexa e paraboloide convexo. Note que, quanto mais concentrada no centro for a demanda, maior será a magnitude da força atrativa, e vice-versa. Além disso, quanto mais distante $\vec{s}$ se encontra do centro do disco, mais a magnitude se aproxima da originada por uma demanda pontual, pois a longas distâncias, com $d_{i}>>R_{i}$, o disco pode ser aproximado por um ponto discreto, seu centro, concentrando toda a demanda. Por fim, quanto mais atrativo for o disco, menor será o custo total associado a este disco, conforme apresentado no gráfico da direita na Figura 6. Observe a convexidade estrita das funções $C_{i}$ quando consideradas demandas não pontuais

Exemplo 3: Na Figura 7 apresentamos as soluções ótimas do modelo de Weber com três regiões circulares de raios $R_{i}=250$, considerando as funções demanda (19)-(26) e os mesmos parâmetros constantes na Tabela 1, com a exceção de que neste caso tomamos como ponto inicial para o método iterativo o ponto médio entre os centros dos discos. O método de Weiszfeld adaptado (13a)-(18) também foi implementado no software matemático MATLAB ${ }^{\circledR}$, utilizando o comando integral 2 no cálculo das integrais duplas. As cores de cada solução correspondem às mesmas utilizadas na Figura 6, sendo que os pontos circulares correspondem às linhas tracejadas da Figura 6. Na Tabela 3 apresentamos as localizações ótimas e o número de iterações até a convergência do método; na Tabela 4 apresentamos os custos totais mínimos associados às posições 
ótimas. Observe que, exceto pelo caso (A), $w_{1}=w_{2}=w_{3}=1$, quanto mais a demanda estiver concentrada no centro dos discos, mais a localização ótima estará próxima da região com maior demanda, sendo menor o custo total mínimo $\square$
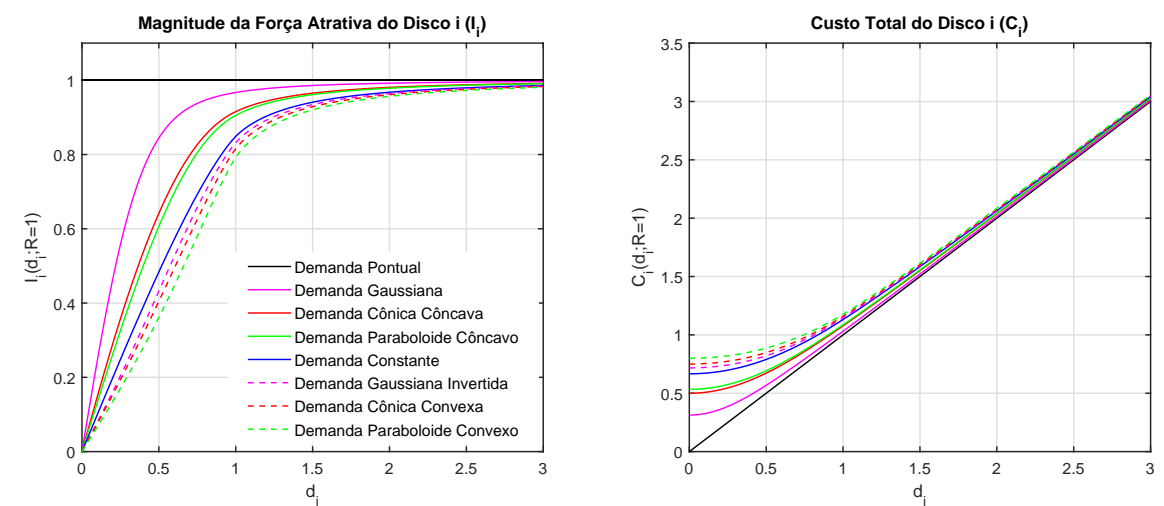

Figura 6 - Força atrativa $I_{i}$ e custo $C_{i}$ das funções demanda unitárias do Exemplo 2 Fonte: Elaborado pelo autor

Tabela 3 - Localização ótima e número de iterações para o problema do Exemplo 3

\begin{tabular}{|c|c|c|c|c|}
\hline & $(\mathrm{A})$ & $(\mathrm{B})$ & $(\mathrm{C})$ & $(\mathrm{D})$ \\
\hline Demanda/Custo Total & $\vec{s}^{*} ;$ Iterações & $\vec{s}^{*} ;$ Iterações & $\vec{s}^{*} ;$ Iterações & $\vec{s}^{*} ;$ Iterações \\
\hline Pontual & $(500,00 ; 330,94) ; 2$ & $(692,79 ; 219,63) ; 63$ & $(522,51 ; 615,78) ; 102$ & $(339,31 ; 273,48) ; 51$ \\
\hline Gaussiana & $(500,00 ; 330,94) ; 2$ & $(672,19 ; 231,52) ; 47$ & $(529,87 ; 567,63) ; 55$ & $(349,01 ; 281,12) ; 43$ \\
\hline Cônica Côncava & $(500,00 ; 330,94) ; 2$ & $(652,81 ; 242,72) ; 37$ & $(535,42 ; 534,52) ; 40$ & $(359,86 ; 289,70) ; 37$ \\
\hline Paraboloide Côncavo & $(500,00 ; 330,94) ; 2$ & $(649,91 ; 244,39) ; 36$ & $(536,21 ; 530,02) ; 39$ & $(361,61 ; 291,09) ; 36$ \\
\hline Constante & $(500,00 ; 330,94) ; 2$ & $(637,03 ; 251,82) ; 31$ & $(539,67 ; 510,62) ; 33$ & $(369,65 ; 297,41) ; 32$ \\
\hline Gaussiana Invertida & $(500,00 ; 330,94) ; 2$ & $(633,74 ; 253,73) ; 30$ & $(540,52 ; 505,91) ; 32$ & $(371,81 ; 299,10) ; 31$ \\
\hline Cônica Convexa & $(500,00 ; 330,94) ; 2$ & $(630,89 ; 255,37) ; 29$ & $(541,26 ; 501,84) ; 31$ & $(373,68 ; 300,56) ; 30$ \\
\hline Paraboloide Convexo & $(500,00 ; 330,94) ; 2$ & $(627,13 ; 257,54) ; 28$ & $(542,22 ; 496,54) ; 30$ & $(376,19 ; 302,50) ; 29$ \\
\hline
\end{tabular}

Tabela 4 - Custos totais mínimos para o problema do Exemplo 3

\begin{tabular}{|c|c|c|c|c|}
\hline Demanda/Custo Total & $(\mathrm{A})$ & $(\mathrm{B})$ & $(\mathrm{C})$ & $(\mathrm{D})$ \\
\hline Pontual & $1.385,64$ & $1.568,38$ & $1.982,41$ & $2.706,57$ \\
\hline Gaussiana & $1.385,64$ & $1.568,80$ & $1.984,09$ & $2.706,80$ \\
\hline Cônica Côncava & $1.385,64$ & $1.570,02$ & $1.987,39$ & $2.707,61$ \\
\hline Paraboloide Côncavo & $1.385,64$ & $1.570,27$ & $1.987,99$ & $2.707,80$ \\
\hline Constante & $1.385,64$ & $1.571,64$ & $1.991,00$ & $2.708,86$ \\
\hline Gaussiana Invertida & $1.385,64$ & $1.572,05$ & $1.991,84$ & $2.709,21$ \\
\hline Cônica Convexa & $1.385,64$ & $1.572,43$ & $1.992,61$ & $2.709,53$ \\
\hline Paraboloide Convexo & $1.385,64$ & $1.572,96$ & $1.993,65$ & $2.709,99$ \\
\hline
\end{tabular}

Exemplo 4: Neste exemplo consideramos dez regiões circulares com centros, raios e demandas totais diversas, com várias destas regiões se sobrepondo umas às outras. Os parâmetros detalhados deste problema são apresentados na Tabela 5. Os resultados para as funções demanda 
(A)

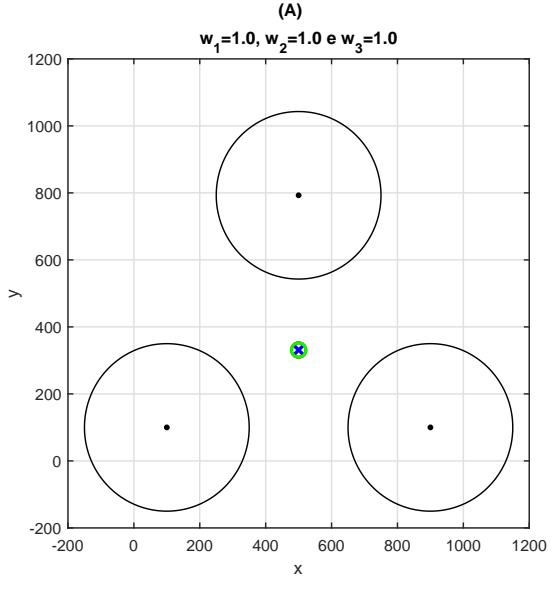

(C)

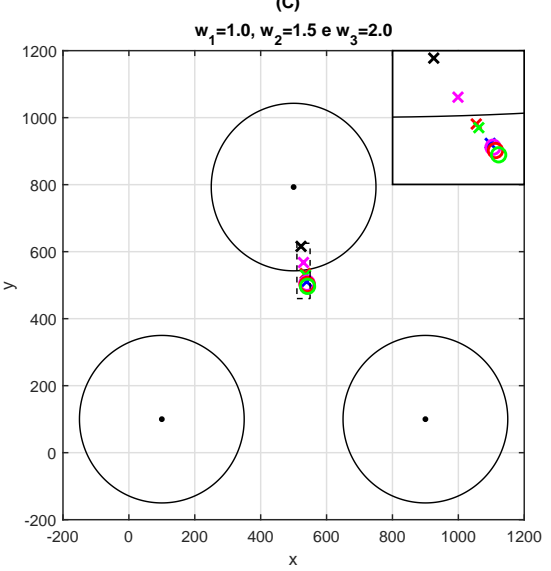

(B)
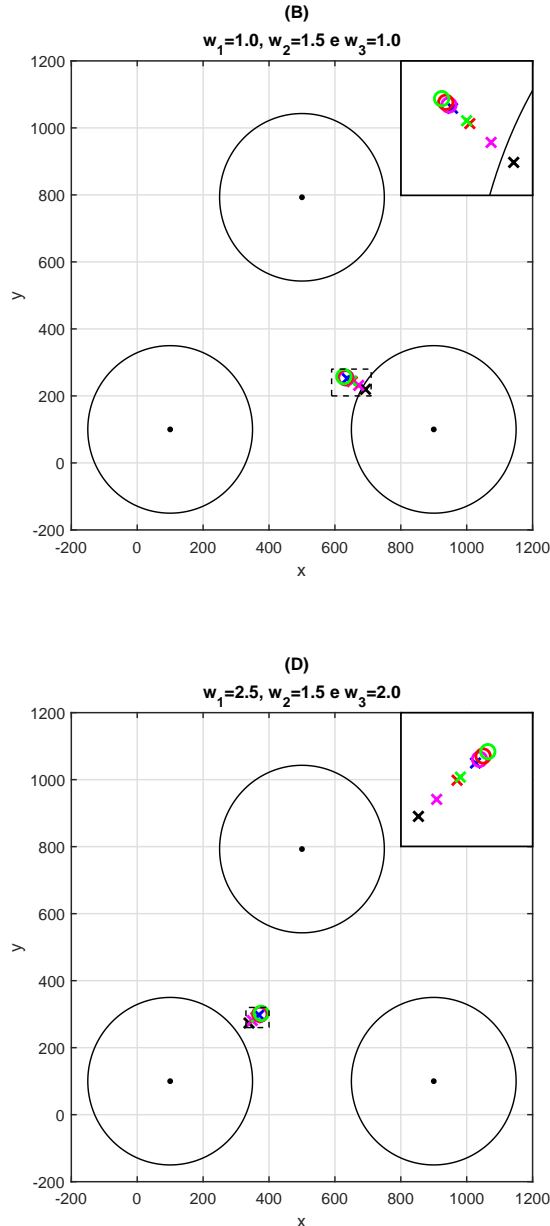

Figura 7 - Solução do modelo de Weber com demandas circulares $n=3, R_{i}=250, w_{i}=1$ (Exemplo 3)

Fonte: Elaborado pelo autor

(19)-(26) são apresentados na Tabela 6 e na Figura 8. Como podemos ver, o método (13a)-(18) converge em menos de 20 iterações para todas as demandas consideradas. Além disso, novamente observamos que quanto mais as demandas estiverem concentradas no centro dos discos, menor será o custo total mínimo $\square$

\section{Conclusões}

Neste artigo consideramos o problema de localização industrial de Weber. Inicialmente apresentamos uma breve revisão do problema original, considerando uma firma e fornecedores/mercados pontuais, derivamos o método iterativo de Weiszfeld, o qual foi desenvolvido para sua solução numérica, e o aplicamos em um exemplo com três fornecedores/mercados dados. Em seguida, apresentamos uma generalização do problema original, onde consideramos uma firma 
Tabela 5 - Parâmetros utilizados no Exemplo 4

\begin{tabular}{|c|c|c|c|}
\hline Região $(i)$ & Centro $\left(\vec{x}_{i}\right)$ & Raio $\left(R_{i}\right)$ & Demanda $\left(w_{i}\right)$ \\
\hline 1 & $(3 ; 0)$ & 0,49 & 4,51 \\
\hline 2 & $(9 ; 0)$ & 2,38 & 0,84 \\
\hline 3 & $(6 ; 5)$ & 0,93 & 2,29 \\
\hline 4 & $(6 ; 8)$ & 1,59 & 9,13 \\
\hline 5 & $(10 ; 10)$ & 0,50 & 1,52 \\
\hline 6 & $(3 ; 1)$ & 1,81 & 8,26 \\
\hline 7 & $(8 ; 6)$ & 0,79 & 5,38 \\
\hline 8 & $(8 ; 5)$ & 1,96 & 9,96 \\
\hline 9 & $(4 ; 0)$ & 2,07 & 0,78 \\
\hline 10 & $(6 ; 3)$ & 2,24 & 4,43 \\
\hline
\end{tabular}

Tabela 6 - Solução ótima do problema do Exemplo 4

\begin{tabular}{|c|c|c|c|}
\hline Demanda/Custo Total & $\vec{s}^{*}$ & Custo Mínimo (\$) & Iterações \\
\hline Pontual & $(6,78 ; 4,89)$ & 151,96 & 19 \\
\hline Gaussiana & $(6,69 ; 4,87)$ & 151,99 & 16 \\
\hline Cônica Côncava & $(6,61 ; 4,85)$ & 152,07 & 15 \\
\hline Paraboloide Côncavo & $(6,59 ; 4,85)$ & 152,08 & 14 \\
\hline Constante & $(6,53 ; 4,85)$ & 152,17 & 14 \\
\hline Gaussiana Invertida & $(6,52 ; 4,85)$ & 152,20 & 14 \\
\hline Cônica Convexa & $(6,51 ; 4,85)$ & 152,23 & 14 \\
\hline Paraboloide Convexo & $(6,58 ; 4,85)$ & 152,27 & 14 \\
\hline
\end{tabular}

pontual e mercados dados por regiões circulares apresentando funções densidades de demanda radialmente simétricas, derivando um método de Weiszfeld modificado para sua solução numérica. A simetria radial das funções demanda permitiu a obtenção de fórmulas integrais simplificadas, em coordenadas polares, para este método iterativo, as quais são uma extensão para uma classe de demandas não constantes das fórmulas obtidas por Chen (2001).

Como exemplos de funções densidade de demanda radialmente simétricas, consideramos: funções onde a demanda está mais concentrada no centro do disco - funções delta de Dirac, gaussiana, cônica côncava e paraboloide côncavo -; a função demanda constante; e funções onde a densidade de demanda está mais concentrada próxima à fronteira do disco - funções paraboloide convexo, cônica convexa e gaussiana invertida.

A solução numérica de um problema de localização com três regiões circulares com raios iguais e demandas diversas, e de um problema com dez regiões circulares com raios, demandas e posições diversas, onde neste último caso há a sobreposição entre várias regiões, considerando as demandas radialmente simétricas acima, obtemos a conclusão de que o método iterativo converge em relativamente poucas iterações para a posição ótima (em termos temporais a execução do método não passou de uns poucos segundos em um computador com configurações medianas), e que, quanto mais concentrada no centro do disco estiver a demanda, menor é o custo total mínimo obtido.

O modelo de localização tratado aqui apresenta as seguintes limitações na resolução de problemas práticos: na prática, a distância entre dois pontos não é necessariamente dada por uma reta os conectando, mas geralmente pelo comprimento de rotas alternativas descrevendo curvas mais complexas no plano, as quais são definidas pela estrutura viária existente; no geral, as regiões 


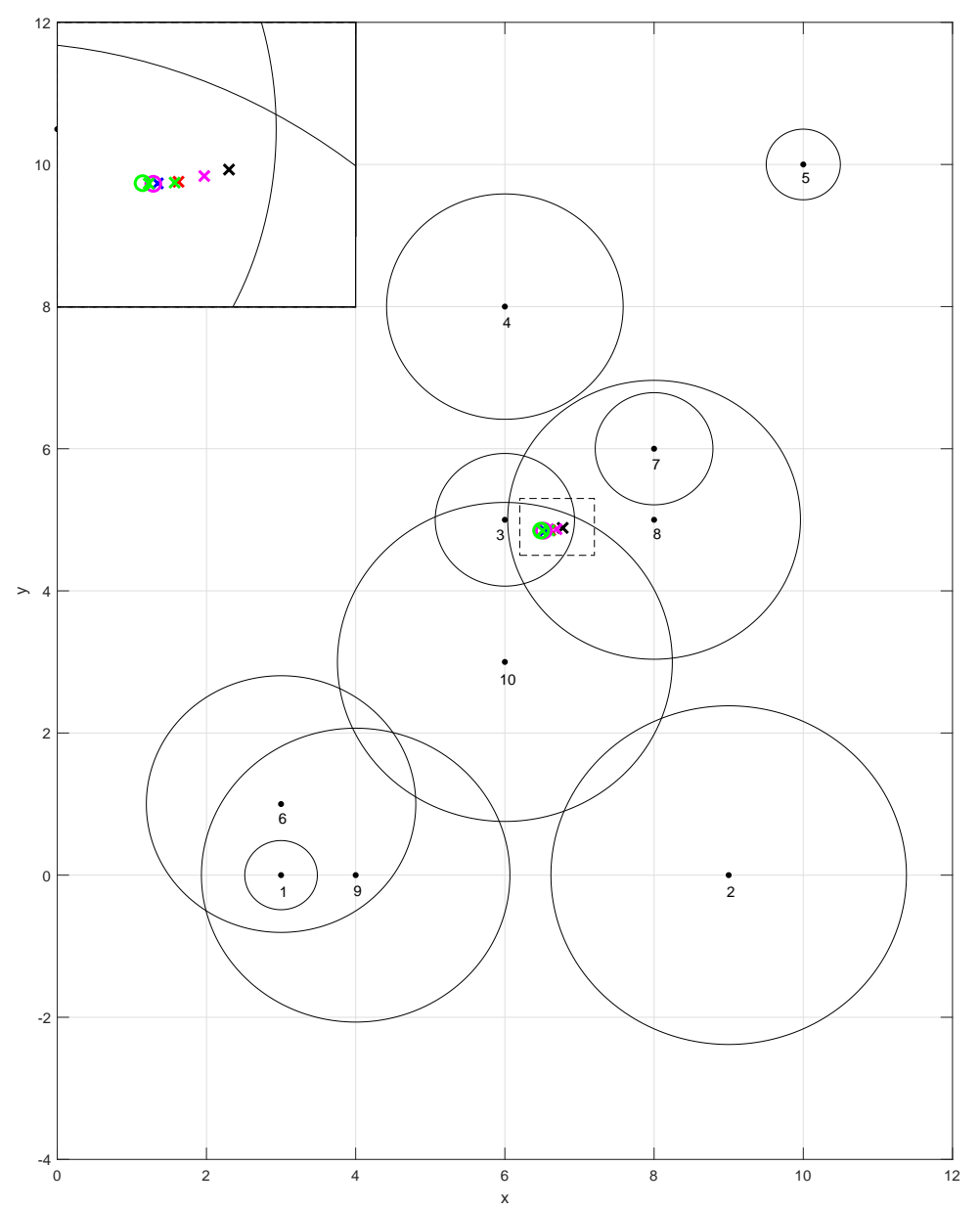

Figura 8 - Solução do modelo de Weber com demandas circulares do Exemplo 4 Fonte: Elaborado pelo autor

de demanda não são perfeitamente circulares; e por fim, as funções de demanda usualmente não são radialmente simétricas. O tratamento adequado de tais limitações constituem tópicos para pesquisas futuras.

\section{Referências bibliográficas}

BECKMANN, M. J.; THISSE, J. P. The location of production activities. In: NIJKAMP, P. (org.). Handbook of regional and urban economics: regional economics. Amsterdam: Elsevier Science Publishers, 1986. v. 1, p. 21-95.

BRIMBERG, J. Further notes on convergence of the Weiszfeld algorithm. Yugoslav Journal of Operations Research, v. 13, n. 2, p. 199-206, 2003. 
CARRIZOSA, E.; CONDE, E.; MUÑOZ-MARQUEZ, M.; PUERTO, J. The generalized Weber problem with expected distances. Revue française d'automatique, d'informatique et de recherche opérationnelle - Recherche opérationnelle, v. 29, n. 1, p. 35-57, 1995.

CHEN, R. Optimal location of a single facility with circular demand areas. Computers \& Mathematics with Applications, v. 41, n. 8, p. 1049-1061, 2001.

DREZNER, Z. Location of regional facilities. Naval Research Logistic Quarterly, v. 33, n. 3, p. 523-529, 1986.

KOSHIZUKA, T.; KURITA, O. Approximate formulas of average distances associated with regions and their applications to location problems. Mathematical Programming, v. 52, n. 1, p. 99-123, 1991.

KUHN, H. W. On a pair of dual nonlinear programs. In: ABADIE, J. (ed.). Nonlinear Programming. Amsterdam: Noth-Holland Publishing Company, 1967. p. 37-54.

KUHN, H. W. A note on Fermat's problem. Mathematical Programming, v. 4, n.1, p. 98-107, 1973.

LOVE, R. F. A computational procedure for optimally locating a facility with respect to several rectangular regions. Journal of Regional Science, v. 12, n. 2, p. 233-242, 1972.

MCCANN, P. Modern urban and regional economics. 2nd. ed. Oxford: Oxford University Press, 2013.

WEBER, A. The theory of the location of industries. Chicago: Chicago University Press, 1929.

WEISZFELD, E. Sur le point pour lequel la somme des distances de n points donnés est minimum. Tôhoku Mathematics Journal, v. 43, p. 355-386, 1937. 